\title{
Surface-field cavity based on a two-dimensional cylindrical lattice
}

\author{
I. V. Konoplev, ${ }^{1, a)}$ L. Fisher, ${ }^{1}$ K. Ronald, ${ }^{1}$ A. W. Cross, ${ }^{1}$ A. D. R. Phelps,${ }^{1}$ C. W. Robertson, ${ }^{1}$ \\ and $\mathrm{M}$. Thumm ${ }^{2}$ \\ ${ }^{1}$ Department of Physics, SUPA, University of Strathclyde, Glasgow G4 ONG, United Kingdom \\ ${ }^{2}$ Institute for Pulsed Power and Microwave Technology (IHM) and Institute of High Frequency Techniques \\ and Electronics (IHE), Karlsruhe Institute of Technology (KIT), Karlsruhe D-76131, Germany
}

(Received 26 March 2010; accepted 21 April 2010; published online 9 June 2010)

The results of theoretical and experimental studies of a high-Q cavity based on a cylindrical, periodic lattice are presented. The coupling of localized surface and volume electromagnetic fields results in cavity mode selection over radial, azimuthal, and longitudinal indices and formation of a high-Q cavity eigenmode. Numerical analyses of the field evolution inside the cavity were carried out. Application of these two-dimensional periodic structures in the development of high-power terahertz masers is proposed. (c) 2010 American Institute of Physics. [doi:10.1063/1.3428776]

Understanding the properties of electromagnetic fields within periodic structures that are oversized in comparison with the radiation wavelength is an important and challenging problem for modern science and such studies have already resulted in the development of breakthrough ideas. ${ }^{1-8}$ For example in 2003 a concept of a surface plasmon amplifier was introduced and is an ongoing area of research. ${ }^{7-11}$ Let us note that in the works ${ }^{7-10}$ nanoshells and nanowires were used to form the interaction region, with localized fields tightly coupled to the surface of the particles. In our work we consider the "inverted model" of the structures studied in Refs. 7-10, i.e., surface fields inside the structure are investigated and are coupled to the corrugated inner surface of the cylindrical metal waveguide. The structure studied was made from an oversized (in comparison with an operating wavelength $\lambda$ ) cylindrical, copper waveguide with a two-dimensional (2D) corrugation of the inner wall. The corrugation supports localized surface fields mitigating the field bonding to the metal surface of the waveguide. The structure is a high-Q cavity in which excitation takes place in three stages. In the first stage the excitation of surface currents around an elementary cell of the periodic surface by the incident electromagnetic field occurs. Then the inductively coupled surface currents are interacting collectively ensuring excitation of a coherent surface field (decaying toward the center of the structure). In the third stage the energy stored in the surface fields is coupled into the propagating waves, which ensures mode selectivity due to scattering and diffraction allowing a high-Q cavity to be observed. In this paper the coupling between localized surface (having imaginary transverse wavenumber) and volume (having real transverse wavenumber) fields on the surface of the cylindrical 2D periodic lattice ${ }^{12-15}$ is studied and the results observed are discussed. The coupling of the localized surface fields to a near cut-off wave is demonstrated and excitation of a high-Q cavity eigenmode due to such a coupling is investigated. We suggest that implementation of such a cavity will improve the efficiency and stability of masers operating in the terahertz $(\mathrm{THz})$ frequency range. The limitations of such structures and ways to overcome these limits are discussed.

\footnotetext{
${ }^{\text {a) }}$ Author to whom correspondence should be addressed. Electronic mail: ivan.konoplev@strath.ac.uk. Tel.: +44(0)-141-548-5818. FAX: +44-141$552-2891$.
}

The surface wave cavity (Fig. 1) can be formed for instance from a cylindrical copper waveguide by machining small periodic perturbations on the inside of the wall $(\Delta r \ll \lambda)$ such that: $r=r_{0}+\Delta r \cos \bar{k}_{z} z \cos \bar{m} \phi$. Here, $r_{0}$ is the mean radius of the unperturbed waveguide, $\bar{k}_{z}=2 \pi / d_{z}$ is the longitudinal wave number of the structure, and $\bar{m}$ is the number of azimuthal variations around the structure. The structure was constructed using electrochemical copper deposition on an aluminum mandrel which was dissolved later in alkaline solution. Let us note that a large diameter structure such that $r_{0} \gg d_{z}$ and $r_{0} \gg \lambda$ is studied in the experiment allowing a "square wave," chessboard structure to be used in the numerical model as an approximation ${ }^{12-15}$ of the lattice shown in Fig. 1. The field of the cavity eigenmode outside the periodic corrugation (i.e., $r \leq r_{0}-\Delta r$ ) can be represented as a superposition of surface and volume (subscripts " $s$ " and " $v ")$ partial fields the transverse structures of which coincide with the transverse structures of the eigenmodes of the unperturbed cylindrical waveguide. ${ }^{12-15}$ The surface fields have imaginary transverse wavenumbers while the volume, near cut-off, fields have zero longitudinal and real transverse wavenumbers. The transverse structure of the surface fields can be described as a superposition of $E$ and $H$

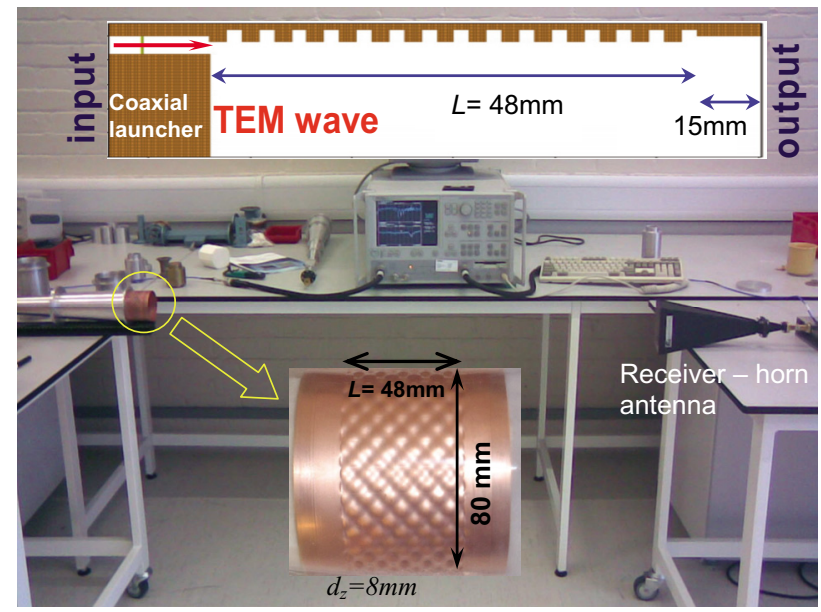

FIG. 1. (Color online) Photograph of the experimental setup and cylindrical 2D periodic structure (inset) which forms the cavity having diameter $80 \mathrm{~mm}$, corrugation depth $1 \mathrm{~mm}, 28$ azimuthal variations and longitudinal period of $8 \mathrm{~mm}$. 
surface modes and taking into account the periodicity of the structure the Fourier field decomposition can be applied:

$$
\left(\frac{E_{z}}{H_{z}}\right)=I_{m_{s}}\left(k_{\perp s} r\right)\left(\begin{array}{c}
\sin m_{s} \varphi \\
\cos m_{s} \varphi
\end{array}\right) \sum_{n=-\infty}^{\infty}\left(\begin{array}{l}
E_{n}(z) \\
H_{n}(z)
\end{array}\right) e^{i n \bar{k}_{z} z}
$$

where $E_{z}, H_{z}$ are the longitudinal field components, $n$ is the harmonic number, $k_{\perp \mathrm{s}}$ is the transverse wave number, $I_{m}(x)$ is the modified Bessel function of order $m$ indicating field decay along $x$, and $E_{n}(z)$ and $H_{n}(z)$ are the slowly varying amplitudes of the field harmonics. The surface and volume field coupling takes place on the surface of the lattice allowing the approximate analysis of the cavity behavior, without the need for detailed analysis of the surface field inside the corrugation, which has a more complex structure and its study is outside the scope of this paper. The surface wave is coupled to the near cut-off $\mathrm{TM}_{0 l}$ volume wave described by $E_{z}=J_{0}\left(k_{\perp v} r\right) \sum_{n=-\infty}^{\infty} E_{n}^{v}(z) e^{i n \bar{k}_{z} z}$ where $l$ is the number of radial variations, $k_{\perp v}$ is the transverse wavenumber, and $J_{m}(x)$ is the Bessel function of order $m$. The resonant coupling between partial fields takes place if the Bragg resonance condition $\vec{k}=\vec{k}_{s}-\vec{k}_{v}$ is satisfied, where $\vec{k}$ is the lattice reciprocal vector, and $\vec{k}_{s, v}$ are the surface " $s$ " and volume " $v$ " wave vectors. As a result one notes that a volume wave with zero azimuthal variation and zero longitudinal wave vector $\left(k_{z v}\right.$ $\cong 0$, near cut-off wave) will be excited, if $k_{z s}=\bar{k}_{z}$ (first harmonic) and the number of the surface field azimuthal variations is $m_{s}=\bar{m}$. The volume field will have $l$ radial variations such that $k_{\perp v}=\chi_{l}^{v} / r_{0}$ where $\chi_{l}^{v}$ is the $l^{\text {th }}$ root of the Bessel function of zero order and the frequency of the cavity eigenmodes $\omega_{r}$ having the highest Q-factor $\left(k_{z v} \cong 0\right)$ can be estimated as $\omega_{r}=c k_{\perp v}$. Taking into account that $k_{\perp s}=i \sqrt{(\omega / c)^{2}-k_{z s}^{2}}$ and $\bar{k}_{z} \geq \omega / c$ one notes that the first harmonic of the surface wave is decaying spatially from the periodic surface as $I_{\bar{m}}\left(k_{\perp s} r_{0}\right)$ demonstrating one of the conventional $^{2}$ surface structure's limitations to creating a large aperture $\left(k_{\perp s} r_{0} \gg 1\right)$ Cherenkov maser which is driven by an electron beam. The field decay results in a weak coupling between the field and the electron beam if $2 k_{\perp s} r_{0} \leq 1$ is not satisfied. To overcome this limit, relativistic electron beams are used in conventional devices ${ }^{2}$ leading to $\bar{k}_{z}$ $\rightarrow \omega / c$ and thus $k_{\perp s} r_{0} \rightarrow 0$. In the structure investigated the coupling of the surface fields and near cut-off propagating wave allows electromagnetic energy accumulation ${ }^{16}$ thus relaxing the condition on the beam-lattice proximity. To illustrate this one may introduce an effective surface at a radius, $r_{\mathrm{e}}$, on which the impedances of the partial waves are matched and $r_{\mathrm{e}}$ can be defined by the equation:

$$
k_{\perp s}\left[I_{\bar{m}}\left(k_{\perp s} r_{\mathrm{e}}\right) / I_{\bar{m}}^{\prime}\left(k_{\perp s} r_{\mathrm{e}}\right)\right]=k_{\perp v}\left[J_{0}\left(k_{\perp v} r_{\mathrm{e}}\right) / J_{0}^{\prime}\left(k_{\perp v} r_{\mathrm{e}}\right)\right],
$$

where $I_{\bar{m}}^{\prime}(x)$ and $J_{0}^{\prime}(x)$ are the derivatives $(\partial / \partial x)$ of the Bessel functions. The $r_{\mathrm{e}}$ defines the beam radius at which it is still interacting with the surface field harmonic. Assuming that $r_{\mathrm{e}} / r_{0} \sim 1$ and applying a Taylor expansion to Eq. (2) the condition under which the electron beam having a relativistic Lorentz factor $\gamma>1.41$, propagating above the lattice can interact with the surface wave takes the form: $k_{\perp s} r_{0}<\bar{m}$, instead of $2 k_{\perp s} r_{0} \leq 1$ for conventional structures.

The finite-difference time-domain (FDTD) threedimensional (3D) code MAGIC has been used to calculate the fields in the cavity. In Fig. 2(a) the structures of the $E_{z}$ and $B_{z}$
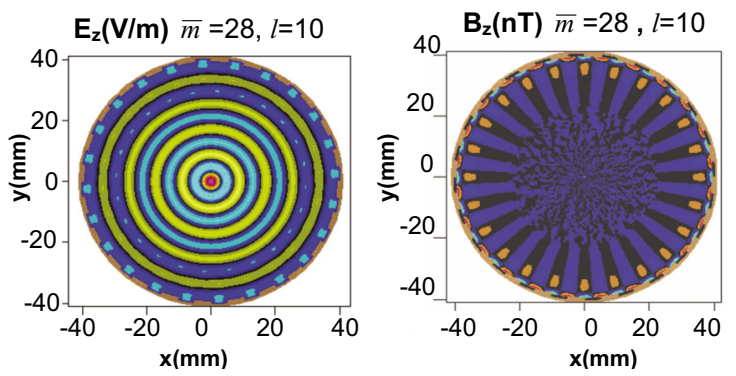

(a)

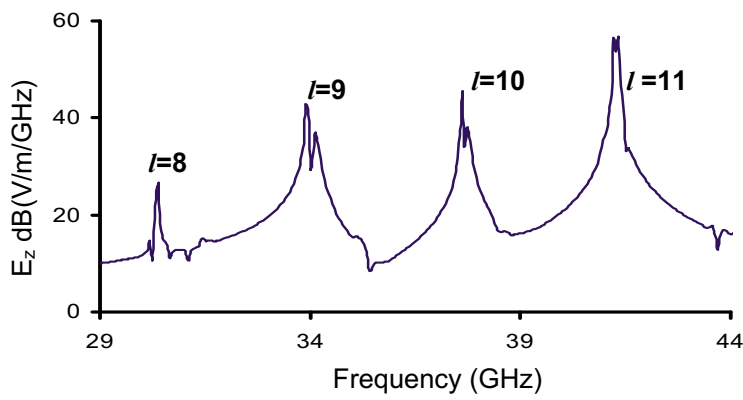

(b)

FIG. 2. (Color online) (a) The $r-\varphi$ contour plot of the $E_{z}$ and $B_{z}$ field components of the cavity eigenmode. The coupling between the surface and volume field can be seen on the first plot while the second plot shows the surface field decaying toward the cavity center (the plot observed using 3D code MAGIC). (b) Spectra of the cavity eigenmodes (with radial indices $l$ $\in[8-11])$ excited by a broadband $[29-44 \mathrm{GHz}]$ pulse formed by the TEM wave.

field components associated with the cavity eigenmode having ten radial variations and located at the frequency $f_{28,10,1}(\sim 37.5 \mathrm{GHz})$ are shown. One notes that only the $E_{z}$ component exists in both the surface and volume partial field while the $B_{z}$ component vanishes toward the center. The spectrum of the cavity eigenmodes excited by a broadband pulse (29-44 GHz) launched in a transverse-electromagnetic (TEM) wave from a coaxial coupler shown in Fig. 1 and calculated using the code MAGIC, is shown in Fig. 2(b). The cavity mode frequencies $f_{m, l, j}(m, l$, and $j$ are the highest variation numbers of volume and surface partial fields along the $\varphi, r$, and $z$ coordinates, respectively) are observed at the frequencies, $f_{28,8,1}(\sim 30 \mathrm{GHz}), f_{28,9,1}(\sim 33.8 \mathrm{GHz}), f_{28,10,1}$ $(\sim 37.5 \mathrm{GHz})$, and $f_{28,11,1}(\sim 41.2 \mathrm{GHz})$, indicating that increasing the operating frequency results in an increase in the eigenmode radial wavenumber.

To study the 2D surface wave cavity experimentally a set up similar to one used for studying coaxial structures ${ }^{14}$ has been constructed. A photograph of the set up with an insert showing the structure studied is presented in Fig. 1. The periodic structure [inset to Fig. 1] made of copper has the following parameters: $r_{0}=40 \mathrm{~mm}$, lattice length $48 \mathrm{~mm}, \bar{m}$ $=28, d_{z}=8 \mathrm{~mm}$, and $\Delta r=0.5 \mathrm{~mm}$. The experiments were carried out using a vector network analyzer (Fig. 1) with a receiving horn antenna positioned at $\sim 1.2 \mathrm{~m}$ distance from the radiating structure. The results observed are shown in Fig. 3. First the receiving detector was positioned centrally (in front of the structure) in the far-field zone and a frequency scan [36-38.5 GHz] was conducted. In Fig. 3(a) the result observed (bold line) is shown in comparison to the same scan performed for a smooth cylindrical waveguide (thin line) of equal length and radius but having no corrugations on the inner surface. The deep minimum in the transmission spectrum is associated with the resonant scattering 

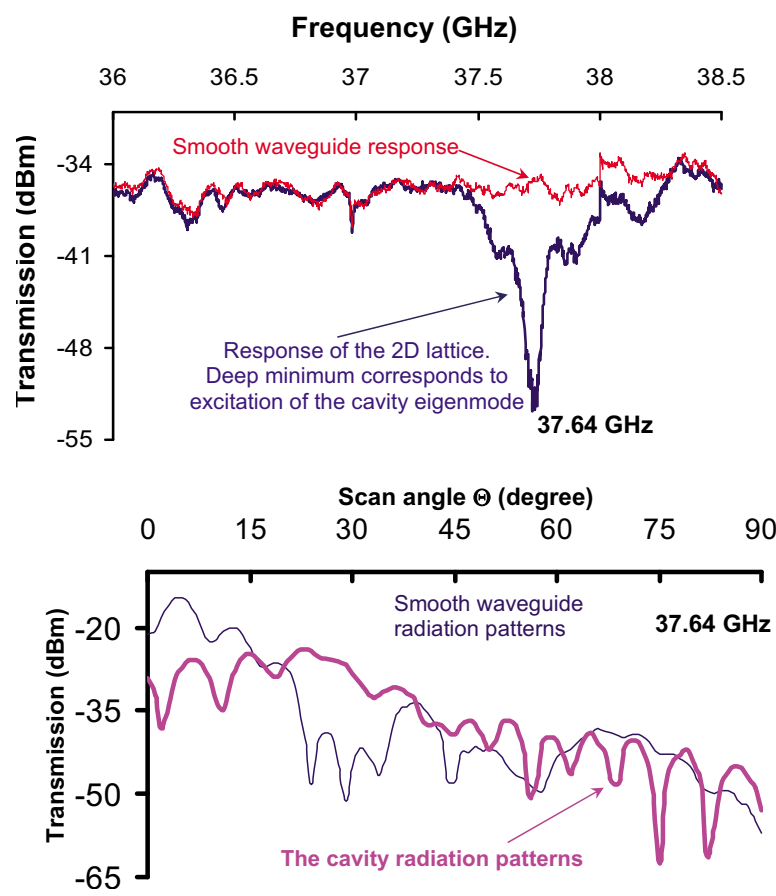

(a)

FIG. 3. (Color online) (a) Frequency dependence of the signal transmission through the smooth waveguide (thin line) and the surface field cavity (thick line) measured by the receiving horn antenna at $1.2 \mathrm{~m}$ distance from the output [see Fig. 1]. (b) The results of the radiation mode pattern measurements in the far field zone $(\sim 1.2 \mathrm{~m})$ from the cavity (thick line) and cylindrical waveguide (thin line).

of the incident wave into surface waves and a near cut-off mode, i.e., excitation of the cavity eigenmode. The results presented resemble those demonstrated in the work of Seymour et al. ${ }^{17}$ and Zayats et al. ${ }^{18}$ where a similar feature in the reflection versus incident angle was reported when surface waves were excited on the metal-dielectric interface. In the case studied in this paper due to a fixed value of the transverse wavenumber (for a specific mode) the frequency variation is similar to the variation in the wave incident angle and the transmission is synonymous with the reflection from the metal-dielectric interface. The excitation of the cavity eigenmode has been also confirmed by analyzing the radiation mode pattern. To conduct the mode pattern scan the horn used in the previous experiments was substituted with a waveguide pick-up probe (to improve the angular resolution). The results of the experimental measurements are shown in Fig. 3(b) (thick line) and compared with the scan when the lattice is substituted with a smooth cylindrical waveguide (thin line). The thin line shows that the radiation from the smooth waveguide consists mainly of low order modes (most of the energy is inside a small solid angle) with a small presence of high order modes. The cavity radiation mode patterns indicate the strong presence of the near cutoff, high-order mode manifesting itself as periodic variations (periodicity $\sim 6^{\circ}$ ) of the field intensity for observation angles above $45^{\circ}$ and much smaller radiation intensity at observation angles below $45^{\circ}$.

In this letter the inverted model of the interaction regions considered in earlier work ${ }^{7-10}$ has been studied. The application of the 2D cylindrical lattice allows mode selection along the radial and azimuthal coordinates, as well as energy storage in the combined fields of the volume and surface waves. This allows an interaction region to be formed having a transverse dimension (aperture) much larger than the operat- ing wavelength. We have shown numerically, using the 3D code MAGIC, that the resonant coupling between surface waves and near cut-off waves takes place on the periodic structure surface. The full 3D model was used to allow excitation and evolution of the azimuthally symmetric and nonsymmetric fields to be simulated. The cavity excitation and the mode structure were analyzed. Experimental studies of the structure have been conducted and the measurements agree well with the numerical simulations. The radiation mode pattern has been studied and incident wave resonant scattering on the periodic lattice has been demonstrated. A cavity of this nature is compatible with a broad variety of active media, including relativistic electron beams ${ }^{19}$ and plasmas and can be used to ensure the stable operation of high-power terahertz masers. The results obtained provide a strong basis for the concept and design of a high power maser operating in the $\mathrm{THz}$ and infrared frequency ranges using a $2 \mathrm{D}$ periodic lattice.

One of the authors, L. Fisher, thanks the EPSRC for supporting her PhD studentship. The UK Faraday Partnership in High Power rf provided the MAGIC license.

${ }^{1}$ E. Yablonovitch, Phys. Rev. Lett. 58, 2059 (1987).

${ }^{2}$ V. L. Bratman, G. G. Denisov, M. M. Ofitserov, S. D. Korovin, S. D. Polevin, and V. V. Rostov, IEEE Trans. Plasma Sci. 15, 2 (1987).

${ }^{3}$ I. V. Konoplev, P. McGrane, A. D. R. Phelps, A. W. Cross, and K. Ronald, Appl. Phys. Lett. 87, 121104 (2005); I. V. Konoplev, P. McGrane, A. W. Cross, K. Ronald, and A. D. R. Phelps, J. Appl. Phys. 97, 073101 (2005). ${ }^{4}$ I. V. Konoplev, A. W. Cross, P. MacInnes, W. He, A. D. R. Phelps, C. G. Whyte, K. Ronald, and C. W. Robertson, Appl. Phys. Lett. 92, 211501 (2008).

${ }^{5}$ J. B. Pendry, L. Martin-Moreno, and F. J. Garcia-Vidal, Science 305, 847 (2004).

${ }^{6}$ I. V. Konoplev, A. W. Cross, A. D. R. Phelps, W. He, K. Ronald, C. G. Whyte, C. W. Robertson, P. MacInnes, N. S. Ginzburg, N. Y. Peskov, A. S. Sergeev, V. Y. Zaslavsky, and M. Thumm, Phys. Rev. E 76, 056406 (2007)

${ }^{7}$ D. J. Bergman and M. I. Stockman, Phys. Rev. Lett. 90, 027402 (2003).

${ }^{8}$ M. I. Stockman, Nat. Photonics 2, 327 (2008).

${ }^{9}$ S. A. Maier, S. R. Andrews, L. Martin-Moreno, and F. J. Garcia-Vidal, Phys. Rev. Lett. 97, 176805 (2006).

${ }^{10}$ D. E. Chang, A. S. Sorensen, P. R. Hemmer, and M. D. Lukin, Phys. Rev. B 76, 035420 (2007).

${ }^{11}$ N. I. Zheludev, S. L. Prosvirnin, N. Papasimakis, and V. A. Fedotov, Nat. Photonics 2, 351 (2008).

${ }^{12}$ A. W. Cross, I. V. Konoplev, K. Ronald, A. D. R. Phelps, W. He, C. G. Whyte, N. S. Ginzburg, N. Y. Peskov, and A. S. Sergeev, Appl. Phys. Lett. 80, 1517 (2002).

${ }^{13}$ N. S. Ginzburg, N. Y. Peskov, A. S. Sergeev, I. V. Konoplev, A. W. Cross, A. D. R. Phelps, G. R. M. Robb, K. Ronald, W. He, and C. G. Whyte, J. Appl. Phys. 92, 1619 (2002); N. S. Ginzburg, N. Y. Peskov, A. S. Sergeev, A. D. R. Phelps, A. W. Cross, and I. V. Konoplev, Phys. Plasmas 9, 2798 (2002).

${ }^{14}$ A. W. Cross, I. V. Konoplev, A. D. R. Phelps, and K. Ronald, J. Appl Phys. 93, 2208 (2003).

${ }^{15}$ I. V. Konoplev, P. McGrane, W. He, A. W. Cross, A. D. R. Phelps, C. G. Whyte, K. Ronald, and C. W. Robertson, Phys. Rev. Lett. 96, 035002 (2006).

${ }^{16}$ I. V. Konoplev, L. Fisher, A. W. Cross, A. D. R. Phelps, and K. Ronald, I.E.T. Conference on High Power RF Technologies (Issues CP548), London, 2009.

${ }^{17}$ R. J. Seymour, J. J. Krupczak, and G. I. Stegeman, Appl. Phys. Lett. 44, 373 (1984).

${ }^{18}$ A. V. Zayats, I. I. Smolyaninov, and A. A. Maradudin, Phys. Rep. 408 131 (2005).

${ }^{19}$ I. V. Konoplev, A. W. Cross, P. MacInnes, W. He, C. G. Whyte, A. D. R. Phelps, C. W. Robertson, K. Ronald, and A. R. Young, Appl. Phys. Lett. 89, 171503 (2006). 